\title{
Do Children With Constipation Have Increased Risk of Asthma? Real-World Data From a Nationwide Population-Based Cohort Study
}

\begin{abstract}
Yen-Chu Huang ${ }^{1 \dagger}$, Meng-Che $\mathrm{Wu}^{1 \dagger}$, Yu-Hsun Wang ${ }^{2}$ and James Cheng-Chung Wei ${ }^{3,45 *}$
${ }^{1}$ Division of Pediatric Gastroenterology, Children's Medical Center, Taichung Veterans General Hospital, Taichung, Taiwan, ${ }^{2}$ Department of Medical Research, Chung Shan Medical University Hospital, Taichung, Taiwan, ${ }^{3}$ Division of Allergy, Immunology and Rheumatology, Chung Shan Medical University Hospital, Taichung, Taiwan, ${ }^{4}$ College of Medicine, Institute of Medicine, Chung Shan Medical University, Taichung, Taiwan, ${ }^{5}$ Graduate Institute of Integrated Medicine, China Medical University, Taichung, Taiwan
\end{abstract}

Background: Asthma is one of the most burdensome childhood disorders. Growing evidence disclose intestinal dysbiosis may contribute to asthma via the gut-lung axis. Constipation can lead to alteration of the gut microbiota. The clinical impact of constipation on asthma has not been researched. Therefore, we aim to assess whether pediatric constipation influence the risk of developing asthma by a nationwide population-based cohort study.

Methods: We analyzed 10,363 constipated patients and 10,363 individuals without constipation between 1999 and 2013 from Taiwan's National Health Insurance Research Database. Analysis of propensity score was utilized to match age, sex, comorbidities, and medications at a ratio of 1:1. In addition, multiple Cox regression analysis was performed to evaluate the adjusted hazard ratio of asthma. Furthermore, sensitivity tests and a stratified analysis were performed.

Results: After adjustment for age, sex, comorbidities, and medications, constipated patients had a 2.36-fold greater risk of asthma compared to those without constipation [adjusted hazard ratio (aHR): 2.36, 95\% C.I. 2.04-2.73, $p<0.001$ ]. Furthermore, the severity of constipation is associated with an increased risk of asthma; the adjusted hazard ratio was $2.25,2.85$, and 3.44 within $<3,3-12$, and $\geq 12$ times of laxatives prescription within 1 year, respectively $(p<0.001)$.

Conclusion: Constipation was correlated with a significantly increased risk of asthma. Pediatricians should be aware of the possibility of asthma in constipated patients. Further research is warranted to investigate the possible pathological mechanisms of this association.

Keywords: constipation, asthma, longitudinal health insurance database, gut-lung axis, dysbiosis

\section{INTRODUCTION}

Asthma is one of the most burdensome childhood disorders. It has been estimated that more than 9 million children in the United States (1) and more than 272 million people worldwide have asthma (2). Asthma not only causes social, psychological, and economic burdens, but also has a negative impact on quality of life (3). Currently, there is increasing evidence demonstrating an 
association between asthma and constipation (4-7), a common situation affecting children globally and a frequent reason for visits to pediatricians. The mean worldwide prevalence of constipation in children is about $12 \%$ (8). Although constipation rarely leads to life-threatening complications, it is a cause of physical and psychological distress for children and their families, eventually impairing quality of life and leading to increased health insurance costs.

In recent years, constipation has been shown to be a causative factor in bowel dysbiosis and therapeutic administration has been increasingly applied. Such therapies include probiotics, prebiotics, or synbiotics, which are thought to help regulate the intestinal microbiota (9). Moreover, recent research has shown that the gut microbiota exerts important regulatory effects via the gut-lung axis (10). For instance, dysbiosis (11) and a lower concentration of short-chain fatty acids (SCFAs) (12) in the intestines have been observed in patients with asthma. This condition leads to dysregulation of inflammation (13) and defects in the intestinal epithelial barriers, resulting in increased intestinal permeability ("leaky gut"), which permits the penetration of toxins and microbiome into systemic circulation, thereby activating $\mathrm{T}$ helper 2 (Th2) immune responses and then releasing the cytokines such as IL-4, IL-5, IL-9, and IL13 into systemic circulation, eventually contributing to airway inflammation (14). Some research has suggested that allergic diseases, such as allergic rhinitis (15) and atopic dermatitis (16), might be connected to constipation. Furthermore, previous research has demonstrated that fecal stasis over a long period could influence the microflora and intestinal environment, resulting in deleterious effects on mucosal immunity and intestinal motility (17). It has not been conclusively established that constipation predisposes susceptible people to asthma. Currently, there are still scanty data on the association between constipation and asthma in the literature. Moreover, this association has never been investigated using a largescale national longitudinal database. We hypothesized that constipation could influence the risk of asthma in children and tested this hypothesis by analyzing a real-world, populationbased retrospective cohort from the National Health Insurance Research Database (NHIRD) in Taiwan.

\section{METHODS}

\section{Data Source}

This study analyzed data from the National Health Insurance Research Database (NHIRD), which contains the healthcare data of almost 99\% of Taiwan's entire population, i.e., $\sim 23$ million NHI beneficiaries. The database includes all insurance claims data, including outpatient visits, emergency visits, and hospitalizations. The Longitudinal Health Insurance Database, (LHID) is a subset of the NHIRD comprising one million individuals randomly sampled from the 23 million NHI beneficiaries for the period from 1999 to 2013. The patients' data were de-identified prior to release to the researchers, in accordance with privacy protocols. Written consent from study subjects was not required and waived by the Institutional Review Board of Chung Shan Medical University Hospital (IRB no.
CS15134), because the NHIRD comprises de-identified data for research purposes. The study carried out in accordance with IGH-GCP requirements and the essence of Declaration of Helsinki.

\section{Study Group and Outcome Measurement}

The population was composed of patients aged $\leq 18$ years old with newly diagnosed constipation (ICD-9-CM codes $=564.0$ ) from 2000 to 2012. To ensure accuracy of diagnoses, we excluded any diagnosis of constipation before 2,000 and only patients with at least three outpatient visits or one hospitalization were selected for inclusion in the final analysis. The index date of this cohort was set as the first date of diagnosis of constipation. Furthermore, to ensure that all individuals had new-onset asthma, we ruled out any diagnosis of asthma (ICD-9-CM = 493) happening before the index date.

The outcome variable was defined as a diagnosis of asthma with at least three outpatient visits or once hospitalization and a prescription for an anti-asthmatic medication including inhaled corticosteroids (ICSs), short-acting $\beta$-agonists, and systemic corticosteroids within 1 year (18). The patients were followed up until the occurrence of asthma, 31 December 2013, or withdrawal from the National Health Insurance system, whichever occurred first.

\section{Covariates and Matching}

The baseline characteristics were age, sex, urbanization, allergic rhinitis (ICD-9-CM = 477.9), allergic conjunctivitis (ICD-9-CM $=372.05,372.14$ ), atopic dermatitis (ICD-9-CM = 691), and bronchiolitis (ICD-9-CM = 466.1). The degree of urbanization was clustered into urban, suburban, and rural by population density (people $/ \mathrm{km}^{2}$ ), population ratio of people with college or above educational levels, population ratio of elder people over 65 years old, population ratio of people of agriculture workers and the number of physicians per 100,000 people. The comorbidities were defined as occurring within 1 year prior to the index date with at least three outpatient visits or once hospitalization. In addition, corticosteroids, antihistamines, and antibiotics during the study period were included and defined as usage for at least $\geq 30$ days. To evaluate the severity of constipation, we collected additional information on the total numbers of prescriptions for laxatives [anatomical therapeutic chemical (ATC) classification system code A06A], which were given within 1 year after the index date.

Furthermore, propensity score matching based on age, sex, urbanization, allergic rhinitis, allergic conjunctivitis, atopic dermatitis, bronchiolitis, corticosteroids, antihistamines, and antibiotics was applied to balance the heterogeneity of the two groups. The propensity score was a probability that was estimated through logistic regression. The binary variable was the constipation and non-constipation group.

\section{Statistical Analysis}

Comparisons between the constipation group and nonconstipation group were done using absolute standardized differences (ASD). Whenever the ASD was $<0.1$, the characteristics of the two groups were deemed to be similar (19). 


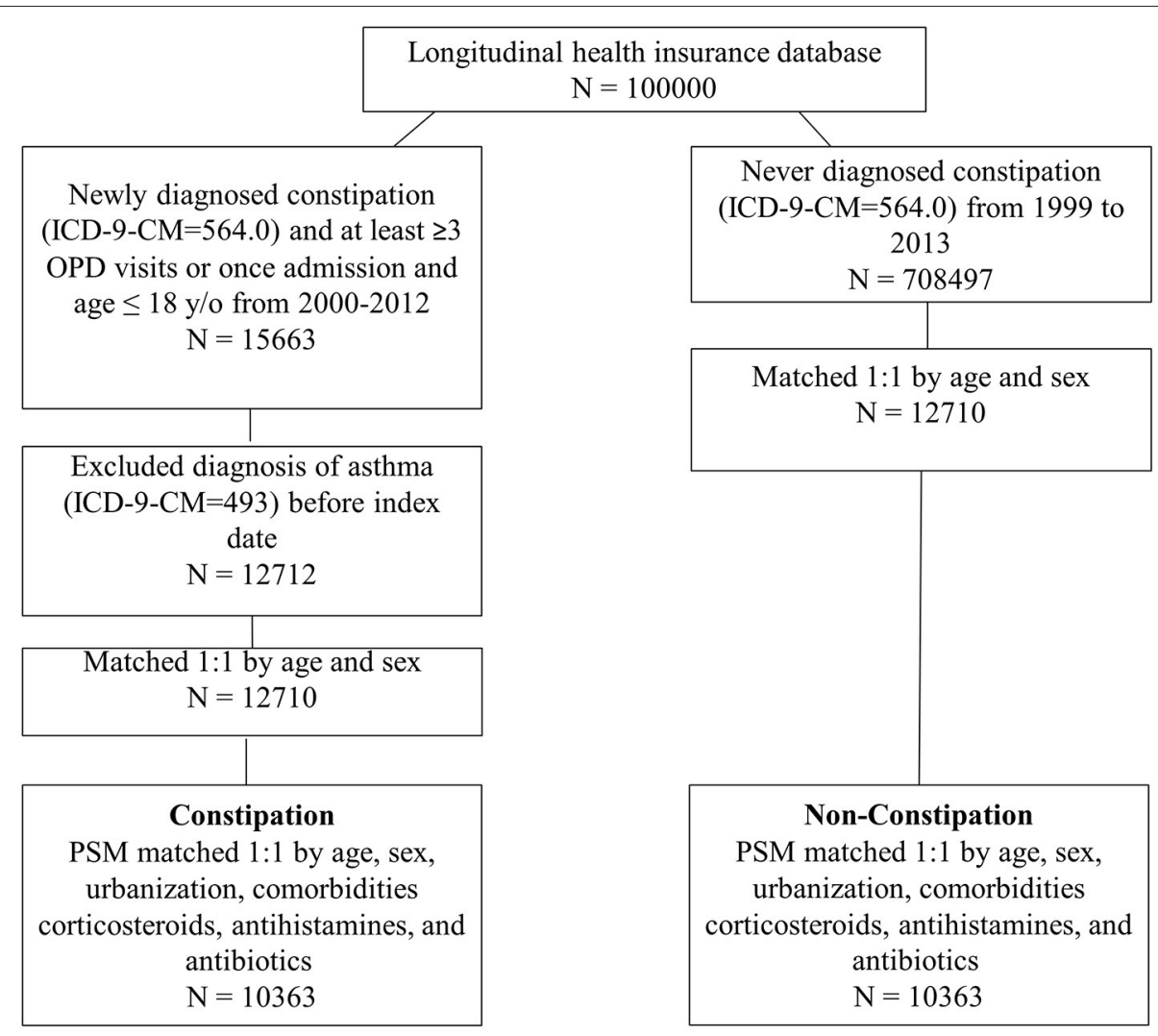

FIGURE 1 | Flowchart of enrolment of constipation and non-constipation groups.

TABLE 1 | Demographic characteristics of the constipation group and non-constipation group.

\begin{tabular}{|c|c|c|c|c|c|c|}
\hline & \multicolumn{2}{|c|}{ Before propensity score matching } & \multirow[b]{2}{*}{ ASD } & \multicolumn{2}{|c|}{ After propensity score matching } & \multirow[b]{2}{*}{ ASD } \\
\hline & Constipation $(N=12,710)$ & Non-constipation $(N=12,710)$ & & Constipation $(N=10,363)$ & Non-constipation $(N=10,363)$ & \\
\hline Age (years) & & & $<0.001$ & & & 0.027 \\
\hline $0-6$ & $4,731(37.2)$ & $4,731(37.2)$ & & $4,157(40.1)$ & $4,064(39.2)$ & \\
\hline $7-12$ & 2,823 (22.2) & 2,823 (22.2) & & 2,206 (21.3) & $2,231(21.5)$ & \\
\hline $13-18$ & $5,156(40.6)$ & $5,156(40.6)$ & & 4,000 (38.6) & 4,068 (39.3) & \\
\hline Mean $\pm \mathrm{SD}$ & $9.4 \pm 5.8$ & $9.4 \pm 5.8$ & $<0.001$ & $9.2 \pm 5.9$ & $9.2 \pm 5.9$ & 0.004 \\
\hline Sex & & & $<0.001$ & & & 0.011 \\
\hline Female & 8,753 (68.9) & $8,753(68.9)$ & & $7,021(67.8)$ & 7,072 (68.2) & \\
\hline Male & 3,957 (31.1) & $3,957(31.1)$ & & 3,342 (32.2) & 3,291 (31.8) & \\
\hline Urbanization & & & 0.081 & & & 0.022 \\
\hline Urban & 6,868 (54.0) & 7,416 (58.3) & & 5,865 (56.6) & $5,801(56.0)$ & \\
\hline Suburban & $4,537(35.7)$ & 4,164 (32.8) & & $3,538(34.1)$ & $3,593(34.7)$ & \\
\hline Rural & 1,305 (10.3) & $1,130(8.9)$ & & $960(9.3)$ & $969(9.4)$ & \\
\hline Allergic rhinitis & $427(3.4)$ & $177(1.4)$ & 0.129 & 205 (2.0) & $177(1.7)$ & 0.020 \\
\hline Allergic conjunctivitis & $121(1.0)$ & $70(0.6)$ & 0.046 & $55(0.5)$ & $62(0.6)$ & 0.009 \\
\hline Atopic dermatitis & $114(0.9)$ & $58(0.5)$ & 0.054 & $44(0.4)$ & $55(0.5)$ & 0.015 \\
\hline Bronchiolitis & $597(4.7)$ & $426(3.4)$ & 0.068 & $414(4.0)$ & $418(4.0)$ & 0.002 \\
\hline Corticosteroids & 2,032 (16.0) & $1,271(10.0)$ & 0.179 & $1,241(12.0)$ & $1,257(12.1)$ & 0.005 \\
\hline Antihistamines & $10,716(84.3)$ & $8,963(70.5)$ & 0.334 & 8,395 (81.0) & $8,379(100.0)$ & 0.004 \\
\hline Antibiotics & 9,790 (77.0) & 7,661 (60.3) & 0.367 & 7,503 (72.4) & 7,442 (71.8) & 0.013 \\
\hline
\end{tabular}

ASD, Absolute standardized differences. 
Cox proportional hazard model was applied to estimate the hazard ratio of asthma between the constipation and nonconstipation groups. Furthermore, Kaplan-Meier analysis was applied to calculate the cumulative incidence of asthma and then log-rank test was used to test the significance.

\section{RESULTS}

The flowchart is shown in Figure 1. We identified 10,363 patients with constipation and 10,363 matched controls between 1999 and 2013 from the LHID. The demographic characteristics of the study participants are shown in Table 1. The individuals in the constipation and non-constipation cohorts were similar in age and sex distribution. After propensity score matching, there were no statistically significant differences between the constipation and non-constipation groups.

Table 2 shows that patients with constipation had a significantly higher risk of asthma than those without constipation after adjustment in the multivariate analysis (aHR: 2.36, 95\% C.I. 2.04-2.73, $p<0.001$ ). Compared with women, men had a significantly higher risk of asthma (aHR: 1.55; 95\% C.I. $1.35-1.77$; $p<0.001$ ). In term of comorbidities, we observed that people with atopic dermatitis or bronchiolitis had a relatively higher risk of asthma (atopic dermatitis:2.18, 95\% C.I. $1.20-3.98, p=0.011$; bronchiolitis:1.42, 95\% C.I.
1.13-1.79, $p=0.003$ ). By contrast, patients using corticosteroids, antihistamines, or antibiotics during the study for a period of at least 30 days had a lower risk of asthma (corticosteroids: 0.79, 95\% C.I. $0.64-0.99, p=0.038$; antihistamines: $0.33,95 \%$ C.I. $0.27-0.40, p<0.001$; antibiotics: 0.38 , 95\% C.I. $0.32-0.44$, $p<0.001)$.

As shown in Table 3, subgroup analyses were performed to assess the association between constipation and asthma based on demographic characteristics. People in the constipation group aged 7-12 years, had a 2.40-fold greater risk of asthma compared with the same age group in the non-constipation group (aHR; 95\% C.I. 1.44-4.01, $P<0.001$ ). Patients aged $0-6$ and $13-18$ years in the constipation group had a 2.22-fold and 1.76-fold greater risk of asthma (aHR; 95\% C.I. 1.89-2.59, $P<0.001$; 95\% C.I. $1.02-3.03 ; P=0.042$ ). Among females, compared with those without constipation, there was a 2.62 -fold higher risk of asthma in patients with constipation (aHR; 95\% C.I. 2.113.26; $P<0.001$ ). Among males, there was 1.89-fold higher risk of asthma in patients with constipation (aHR; 95\% C.I. 1.562.30; $P<0.001$ ). Furthermore, constipated patients had a higher likelihood of asthma, regardless of urbanization, comorbidities and medications.

Table 4 presents the analysis of asthma risk in constipated patients with a prescription of laxatives. Compared to participants without constipation, the adjusted hazard ratio was 2.25-fold (95\% C.I. 1.93-2.61; $P<0.001$ ), 2.85-fold $(95 \%$

TABLE 2 | Cox proportional hazard model analysis for risk of asthma.

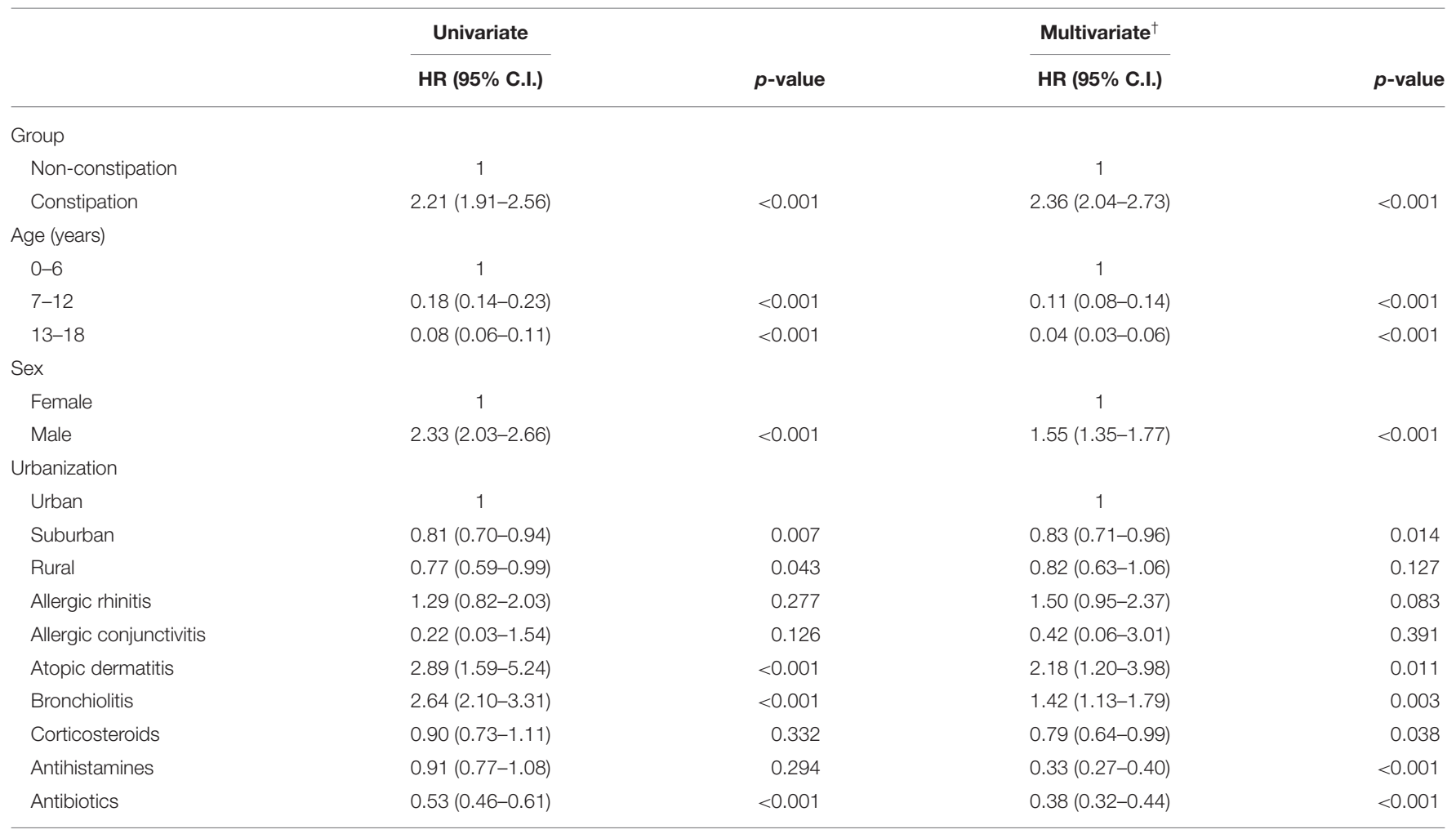

${ }^{\dagger}$ Adjusted for age, sex, urbanization, allergic rhinitis, allergic conjunctivitis, atopic dermatitis, bronchiolitis, corticosteroids, antihistamines, and antibiotics. 
TABLE 3 | Subgroup analysis of the association between constipation and asthma development.

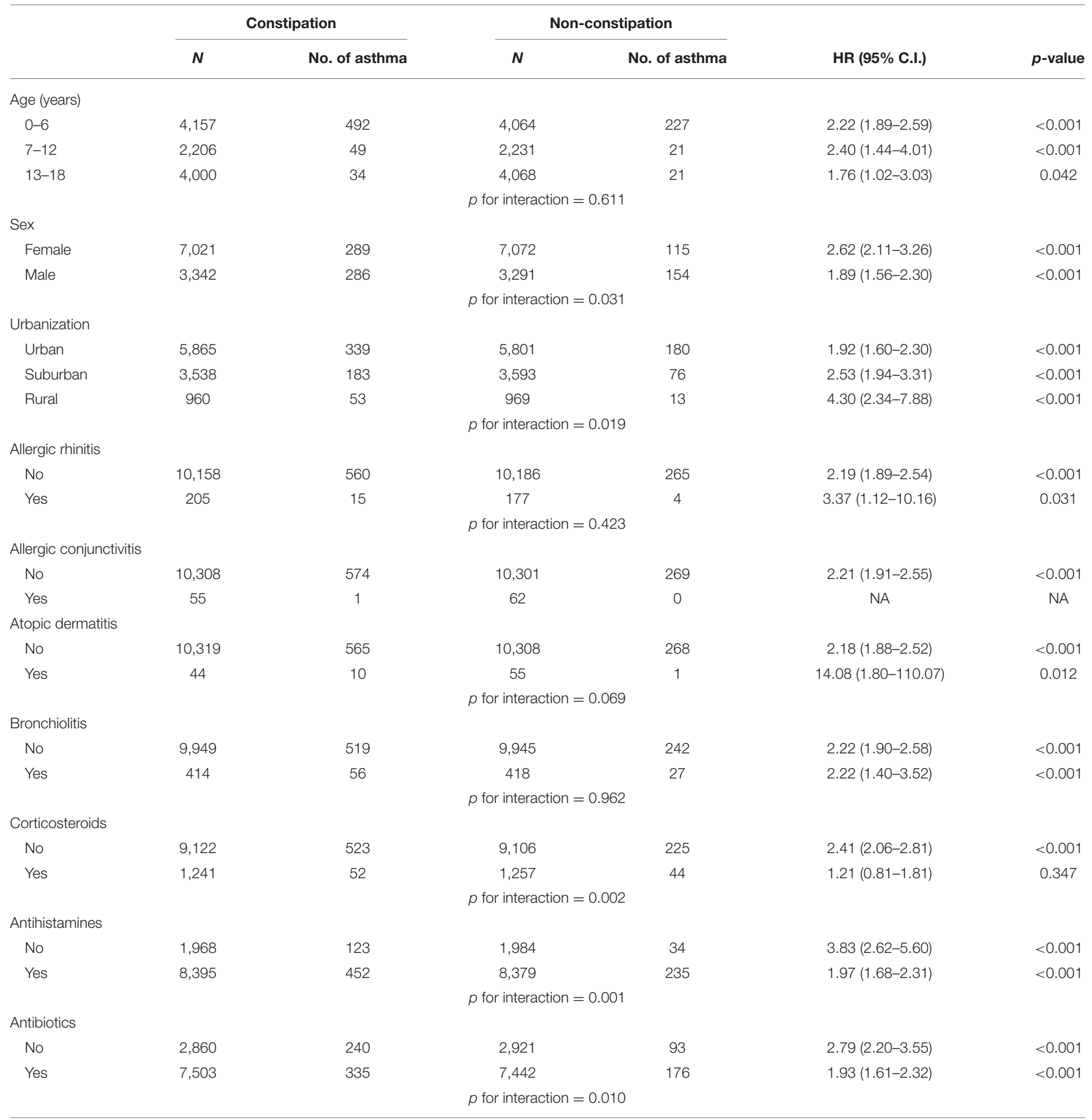

C.I. 2.30-3.53; $P<0.001$ ), and 3.44-fold (95\% C.I. 1.70-6.96; $P<0.001)$ higher risk of developing asthma in constipated patients with $<3,3-12$, and $\geq 12$ times of laxatives prescription within 1 year, respectively. There appeared to be a doseeffect relationship between constipation severity and risk of asthma. The Kaplan-Meier curves are shown in Figure 2. The cumulative incidence of asthma was significantly higher in constipated patients than in non-constipated patients, and the log-rank test for the comparison of cumulative incidence curves resulted in a $P<0.001$.

\section{DISCUSSION}

In this research, children with constipation had a 2.36-fold higher risk for developing asthma than non-constipated individuals, 
TABLE 4 | Cox proportional hazard model analysis for risk of asthma in constipated patients with prescription for laxatives.

\begin{tabular}{|c|c|c|c|c|c|c|}
\hline \multirow[b]{3}{*}{ Group } & \multirow[t]{3}{*}{$N$} & \multirow[t]{3}{*}{ No. of asthma } & \multirow{3}{*}{$\begin{array}{c}\text { Univariate } \\
\text { HR (95\% C.I.) }\end{array}$} & \multirow{3}{*}{$p$-value } & \multicolumn{2}{|l|}{ Multivariate $^{\dagger}$} \\
\hline & & & & & HR (95\% C.I.) & $p$-value \\
\hline & & & & & & \\
\hline Non-constipation & 10,363 & 269 & 1 & & 1 & \\
\hline Constipation with laxatives prescription $<3$ times & 8,557 & 442 & $2.05(1.76-2.39)$ & $<0.001$ & $2.25(1.93-2.61)$ & $<0.001$ \\
\hline Constipation with laxatives prescription $=3-12$ times & 1,709 & 125 & $2.96(2.39-3.66)$ & $<0.001$ & $2.85(2.30-3.53)$ & $<0.001$ \\
\hline Constipation with laxatives prescription $\geq 12$ times & 97 & 8 & $3.45(1.71-6.96)$ & $<0.001$ & $3.44(1.70-6.96)$ & $<0.001$ \\
\hline
\end{tabular}

${ }^{\dagger}$ Adjusted for age, sex, urbanization, allergic rhinitis, allergic conjunctivitis, atopic dermatitis, bronchiolitis, corticosteroids, antihistamines, and antibiotics.

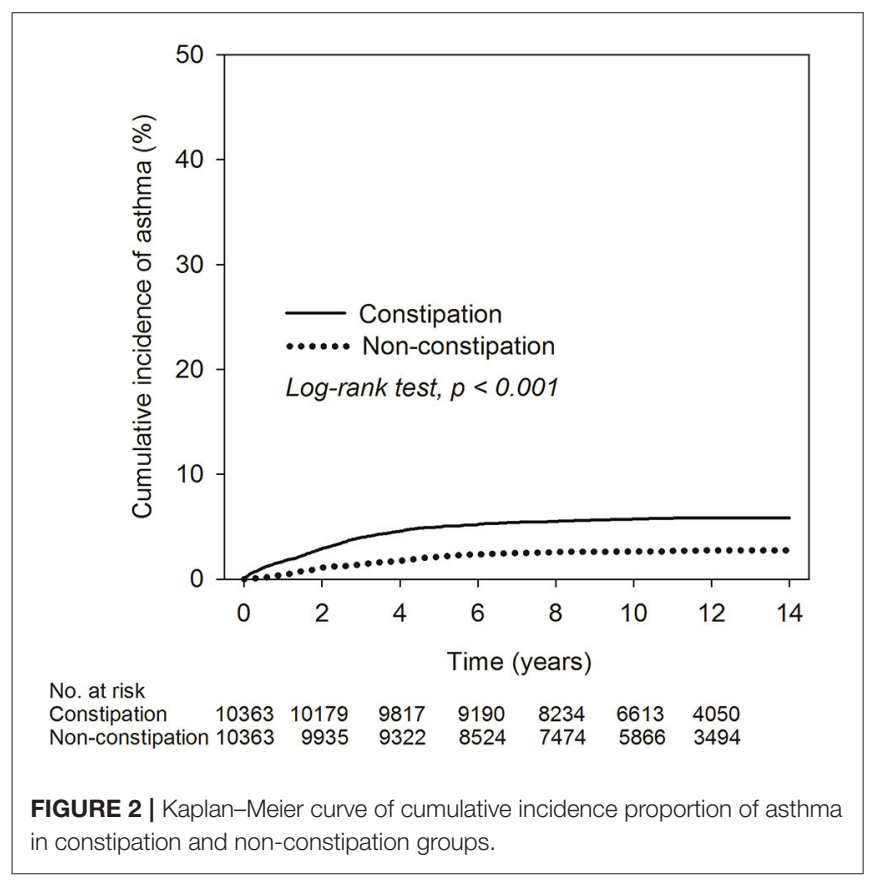

regardless of age, sex, comorbidities, or medications. As far as we know, this is the largest epidemiological study utilizing a nationwide longitudinal population-based dataset to explore the association between constipation and asthma. The findings could be of pathophysiological and clinical importance. Our results highlight the considerably higher risk of asthma in children with constipation. Constipation might be influential in the development of asthma. Pediatricians should be mindful of the possibility of asthma in constipated children. Moreover, constipated patients should be informed of the potential risk of developing asthma and be provided with applicable management for asthma as required. Our findings further highlight the importance of maintaining appropriate bowel habits in order to prevent constipation, which could in turn mitigate risk of asthma.

We also observed that the risk of developing asthma was appreciably increased in children with atopic dermatitis and bronchiolitis. Atopic dermatitis is a chronic inflammatory skin disorder and is regarded as the beginning of the "atopy march" (20), which refers to the natural history of allergic disorders including atopic dermatitis, food allergy (21), asthma, and allergic rhinitis, developing over the course of infancy to childhood. Moreover, some researchers have demonstrated that atopic dermatitis is associated with increased severity of asthma $(22,23)$. In addition, recent studies revealed a connection between developing asthma and bronchiolitis (24). Our study extends these findings as our results indicate that constipation severity is related to risk of asthma. There seemed to be a "dosedependent" relationship between constipation and subsequent risk of asthma, which further supports our hypothesis.

The pathophysiological mechanisms underlying the relationship between constipation and asthma remain ambiguous. Recently, there has been considerable research conducted on the link between asthma and gut microbiota (25). The extended "hygiene hypothesis" posits that the initial composition of the infant intestinal microbiota plays a key part in the development of atopic diseases $(26,27)$. Research conducted in the United Kingdom analyzed the gut microbiome of people with asthma and found that there were abundant Clostridiums spp., whereas Bacteroides stercoris and Faecalibacterium prausnitzii were depleted in individuals with asthma (28). Similarly, some researches have suggested that alterations in intestinal microflora could lead to constipation and constipation-related symptoms (9). Compared to healthy individuals, constipated patients had relatively higher amounts of potentially pathogenic microbes, such as Clostridiums spp. and Pseudomonas aeruginosa, and relatively lower amounts of Lactobacillus, Bacteroides spp., and Bifidobacterium (29). These alterations in the intestinal environment could affect bowel motility by the active metabolites. Some research exhibited that microbial-derived metabolites, predominantly short-chain fatty acids (SCFAs), act as pivotal drivers of T-cell subset activity and proliferation (30). Moreover, it has been found that production of gut microbial SCFAs might down-regulate proinflammatory reactions at the site of allergen insult. Furthermore, SCFAs could affect intestinal motility by stimulating the contraction of colonic smooth muscles, thereby assisting in relief of constipation (31). Another important consideration is the devastating impact of proinflammatory dietary type, or a "Westernized diet," which might be described as being high content of saturated fats, refined carbohydrates, salt, and reduced consumption of vegetables and fruit $(32,33)$, on the immune homeostasis, due to change in the intestinal microbiota, resulting in reduced production of SCFAs. Therefore, low fiber intake, such as a diet with little fresh fruit and vegetables, in children with constipation could 
play a pivotal role in developing asthma (34). The currently available data suggest that the intestinal microbiota might play a key mechanistic role linking asthma and constipation. It is not known how constipation affects the configuration of the bowel microflora and how applicable this condition is to asthma. Nevertheless, constipation seems to be a predisposing factor for asthma. Further comprehensive metabolomic and metagenomic analyses of the intestinal microbiome in constipated children are warranted to clarify the potential mechanisms underlying these associations.

The major advantages of this study were the relatively long follow-up period and the large sample size. An integrated past history of used medical services was accessible for all cases. Therefore, there may have been slight information, selection, and recall bias. As such, it was feasible to properly examine our hypothesis. However, there were still some potential limitations in our study. First, the NHIRD does not include data on covariates, such as mode of delivery, genetic data, personal lifestyle, family history, social adversity, laboratory data, and environmental factors. Even though we adjusted for several comorbidities and matched propensity scores, these unmeasured confounding factors could have influenced our results. Second, the diagnoses of asthma and constipation were dependent on the ICD-9 codes in the administrative dataset. We did not undergo a comprehensive review of the patients' medical records totally so it was not possible to check the accuracy of diagnoses, and thus some misclassifications may have existed. Nevertheless, we tried to increase the accuracy of asthma diagnosis by requiring, in addition to ICD-9 codes, a prescription for an anti-asthmatic medication, as done in the previous studies (35). It is worth noting, however, that any misclassifications were likely to be random, and associations were often underestimated rather than overestimated. In addition, clinical judgment might be different among pediatricians, and so diagnoses would not have been consistent, which might have affected the validity. However, Taiwan's National Health Insurance administration monitors the accuracy of the claims data to prevent violations. Finally, it is unclear as to whether the findings of this study can be extrapolated to other ethnic groups, as the majority of our subjects were Taiwanese. Further clinical research should include other ethnicities and nationalities to determine the generalizability of the relationship observed herein.

In conclusion, constipated children had a 2.36-fold greater risk for asthma compared with those without constipation. Individuals with constipation should be alerted to the

\section{REFERENCES}

1. Gurka MJ, Blackman JA, Heymann PW. Risk of childhood asthma in relation to the timing of early child care exposures. J Pediatr. (2009) 155:7817.e1. doi: 10.1016/j.jpeds.2009.06.035

2. James SL, Abate D, Abate KH, Abay SM, Abbafati C, Abbasi N, et al.Avokpaho FGA, Awasthi A, Ayala Quintanilla BP, Ayer R, Azzopardi PS, Babazadeh A, et al. Global, regional, national incidence, prevalence, and years lived with disability for 354 diseases and injuries for 195 countries and territories, 19902017: a systematic analysis for the global burden of disease study 2017. Lancet. (2018) 392:1789-858. doi: 10.1016/S0140-6736(18)32279-7 elevated risk of developing asthma. Furthermore, in children with asthma, pediatricians should evaluate the patient's bowel condition, including the gut microbiota. The precise pathophysiological association between constipation and asthma requires further research.

\section{DATA AVAILABILITY STATEMENT}

The LHID is a subset of the NHIRD, a database of all medical claims in Taiwan's NHI system. The usage of NHIRD is limited to research purposes only. Only Taiwanese citizens who fulfill the requirements for conducting research projects are eligible to apply for access to the National Health Insurance Research Database (NHIRD). Applicants must follow the Personal Data Protection Act (https://law.moj.gov.tw/ENG/LawClass/LawAll. aspx?pcode=I0050021) and related regulations of the National Health Insurance Administration and NHRI (National Health Research Institutes), and an agreement must be signed by the applicant and his/her supervisor upon application submission. The datasets generated and analyzed during the current study are available from the authors on reasonable request.

\section{ETHICS STATEMENT}

The studies involving human participants were reviewed and approved by Institutional Review Board of Chung Shan Medical University Hospital (Approval number CS15134). Written informed consent to participate in this study was provided by the participants' legal guardian/next of kin. Written informed consent was obtained from the individual(s), and minor(s)' legal guardian/next of kin, for the publication of any potentially identifiable images or data included in this article.

\section{AUTHOR CONTRIBUTIONS}

$\mathrm{Y}-\mathrm{CH}$ and $\mathrm{M}-\mathrm{CW}$ drafted the manuscript. JW revised the manuscript critically. All authors provided a substantial contribution to the conception, design, interpretation of the work, and approved the final version of the manuscript.

\section{FUNDING}

This work was supported by a grant from Taichung Veterans General Hospital Research Foundation (TCVGH-1106501B).

3. Pijnenburg MW, Fleming L. Advances in understanding and reducing the burden of severe asthma in children. Lancet Respir Med. (2020) 8:103244. doi: 10.1016/S2213-2600(20)30399-4

4. Leander M, Cronqvist A, Janson C, Uddenfeldt M, Rask-Andersen A. Healthrelated quality of life predicts onset of asthma in a longitudinal population study. Respir Med. (2009) 103:194-200. doi: 10.1016/j.rmed.2008.09.015

5. Ano S, Morishima Y, Ishii Y, Kawaguchi M, Matsuno Y, Hizawa N. Defecation-related asthma. Intern Med. (2013) 52:685-7. doi: 10.2169/internalmedicine.52.8943

6. Dreher ML. Whole fruits and fruit fiber emerging health effects. Nutrients. (2018) 10:1833. doi: 10.3390/nu10121833 
7. Marpole R, Blackmore AM, Gibson N, Cooper MS, Langdon K, Wilson AC. Evaluation and management of respiratory illness in children with cerebral palsy. Front Pediatr. (2020) 8:333. doi: 10.3389/fped.2020.00333

8. Mugie SM, Benninga MA, Di Lorenzo C. Epidemiology of constipation in children and adults: a systematic review. Best Pract Res Clin Gastroenterol. (2011) 25:3-18. doi: 10.1016/j.bpg.2010.12.010

9. Ohkusa T, Koido S, Nishikawa Y, Sato N. Gut microbiota and chronic constipation: a review and update. Front Med. (2019) 6:19. doi: 10.3389/fmed.2019.00019

10. Dang AT, Marsland BJ. Microbes, metabolites, and the gut-lung axis. Mucosal Immunol. (2019) 12:843-50. doi: 10.1038/s41385-019-0160-6

11. Arrieta MC, Sadarangani M, Brown EM, Russell SL, Nimmo M, Dean J, et al. A humanized microbiota mouse model of ovalbumin-induced lung inflammation. Gut Microbes. (2016) 7:342-52. doi: 10.1080/19490976.2016.1182293

12. Ivashkin V, Zolnikova O, Potskherashvili N, Trukhmanov A, Kokina N, Dzhakhaya N, et al. Metabolic activity of intestinal microflora in patients with bronchial asthma. Clin Pract. (2019) 9:1126. doi: 10.4081/cp.2019.1126

13. Vinolo MA, Rodrigues HG, Nachbar RT, Curi R. Regulation of inflammation by short chain fatty acids. Nutrients. (2011) 3:858-76. doi: 10.3390/nu3100858

14. Barrett NA, Austen KF. Innate cells and $\mathrm{T}$ helper 2 cell immunity in airway inflammation. Immunity. (2009) 31:42537. doi: 10.1016/j.immuni.2009.08.014

15. Wu MC, Jan MS, Chiou JY, Wang YH, Wei JC. Constipation might be associated with risk of allergic rhinitis: a nationwide population-based cohort study. PLoS ONE. (2020) 15:e0239723. doi: 10.1371/journal.pone.0239723

16. Huang Y-C, Wu M-C, Wang Y-H, Wei JC-C. Influence of constipation on atopic dermatitis: a nationwide population-based cohort study in Taiwan. Int J Clin Pract. (2021) 75:e13691. doi: 10.1111/ijcp.13691

17. Zhu L, Liu W, Alkhouri R, Baker RD, Bard JE, Quigley EM, et al. Structural changes in the gut microbiome of constipated patients. Physiol Genomics. (2014) 46:679-86. doi: 10.1152/physiolgenomics.00082.2014

18. Fu LS, Lin CC, Wei CY, Lin CH, Huang YC. Risk of acute exacerbation between acetaminophen ibuprofen in children with asthma. PeerJ. (2019) 7:e6760. doi: $10.7717 /$ peerj.6760

19. Austin PC. Balance diagnostics for comparing the distribution of baseline covariates between treatment groups in propensity-score matched samples. Stat Med. (2009) 28:3083-107. doi: 10.1002/sim.3697

20. Hill DA, Grundmeier RW, Ram G, Spergel JM. The epidemiologic characteristics of healthcare provider-diagnosed eczema, asthma, allergic rhinitis, and food allergy in children: a retrospective cohort study. BMC Pediatr. (2016) 16:133. doi: 10.1186/s12887-016-0673-Z

21. Chen CC, Chen KJ, Kong MS, Chang HJ, Huang JL. Alterations in the gut microbiotas of children with food sensitization in early life. Pediatr Allergy Immunol. (2016) 27:254-62. doi: 10.1111/pai.12522

22. Martinez FD, Wright AL, Taussig LM, Holberg CJ, Halonen M, Morgan WJ. Asthma and wheezing in the first six years of life. The group health medical associates. N Engl J Med. (1995) 332:133-8.

23. Gustafsson D, Sjöberg O, Foucard T. Development of allergies and asthma in infants and young children with atopic dermatitis-a prospective follow-up to 7 years of age. Allergy. (2000) 55:240-5. doi: 10.1034/j.1398-9995.2000.00391.x

24. Thomas AO, Lemanske RF Jr, Jackson DJ. Infections and their role in childhood asthma inception. Pediatr Allergy Immunol. (2014) 25:1228. doi: $10.1111 /$ pai. 12147
25. Zhang D, Li S, Wang N, Tan HY, Zhang Z, Feng Y. The cross-talk between gut microbiota and lungs in common lung diseases. Front Microbiol. (2020) 11:301. doi: 10.3389/fmicb.2020.00301

26. Strachan DP. Hay fever, hygiene, household size. BMJ. (1989) 299:1259-60.

27. Daley D. The evolution of the hygiene hypothesis: the role of earlylife exposures to viruses and microbes and their relationship to asthma and allergic diseases. Curr Opin Allergy Clin Immunol. (2014) 14:3906. doi: 10.1097/ACI.0000000000000101

28. Wang Q, Li F, Liang B, Liang Y, Chen S, Mo X, et al. A metagenome-wide association study of gut microbiota in asthma in UK adults. BMC Microbiol. (2018) 18:114. doi: 10.1186/s12866-018-1257-x

29. Gerritsen J, Smidt H, Rijkers GT, de Vos WM. Intestinal microbiota in human health and disease: the impact of probiotics. Genes Nutr. (2011) 6:209-40. doi: 10.1007/s12263-011-0229-7

30. Smith PM, Howitt MR, Panikov N, Michaud M, Gallini CA, Bohlooly YM, et al. The microbial metabolites, short-chain fatty acids, regulate colonic Treg cell homeostasis. Science. (2013) 341:569-73. doi: 10.1126/science.124 1165

31. Dass NB, John AK, Bassil AK, Crumbley CW, Shehee WR, Maurio FP, et al. The relationship between the effects of short-chain fatty acids on intestinal motility in vitro and GPR43 receptor activation. Neurogastroenterol Motil. (2007) 19:66-74. doi: 10.1111/j.1365-2982.2006.00 853. $\mathrm{x}$

32. Statovci D, Aguilera M, MacSharry J, Melgar S. The impact of western diet and nutrients on the microbiota and immune response at mucosal interfaces. Front Immunol. (2017) 8:838. doi: 10.3389/fimmu.2017. 00838

33. Rizzello F, Spisni E, Giovanardi E, Imbesi V, Salice M, Alvisi P, et al. Implications of the westernized diet in the onset and progression of IBD. Nutrients. (2019) 11:1033. doi: 10.3390/nu11051033

34. Lynch SV. Gut microbiota and allergic disease. New insights. Ann Am Thorac Soc. (2016) 13 (Suppl. 1):S51-4. doi: 10.1513/AnnalsATS.201507-451MG

35. Yoshida S, Ide K, Takeuchi M, Kawakami K. Prenatal and early-life antibiotic use and risk of childhood asthma: a retrospective cohort study. Pediatr Allergy Immunol. (2018) 29:490-5. doi: 10.1111/pai.12902

Conflict of Interest: The authors declare that the research was conducted in the absence of any commercial or financial relationships that could be construed as a potential conflict of interest.

Publisher's Note: All claims expressed in this article are solely those of the authors and do not necessarily represent those of their affiliated organizations, or those of the publisher, the editors and the reviewers. Any product that may be evaluated in this article, or claim that may be made by its manufacturer, is not guaranteed or endorsed by the publisher.

Copyright (C) 2021 Huang, Wu, Wang and Wei. This is an open-access article distributed under the terms of the Creative Commons Attribution License (CC BY). The use, distribution or reproduction in other forums is permitted, provided the original author(s) and the copyright owner(s) are credited and that the original publication in this journal is cited, in accordance with accepted academic practice. No use, distribution or reproduction is permitted which does not comply with these terms. 